\title{
Disease Severity and Social Adjustment of Patients with Obsessive Compulsive Disorder from West Bengal
}

\author{
Rima Das ${ }^{1}$, Souvik Raychaudhuri ${ }^{2}$
}

\section{ABSTRACT}

Obsessive-Compulsive disorder (OCD) is a neuropsychiatric disorder prevalent in population worldwide. In the present study we investigated OCD patients from the state of West Bengal, India. Patients were evaluated for social adjustment using Rosenzweig PF test and severity of OCD using the Yale-Brown Obsessive Compulsive Scale (Y-BOCS). It was found that patients from urban areas had poor social adjustment compared to those from semirural areas. Rural population fared well in adjustment in spite of disease.

Keywords: Obsessive Compulsive Disorder, Y-BOCS , Rosenzweig PF Test, Urban, Rural.

Obsessive Compulsive disorder (OCD) is now viewed as one of the most prevalent psychiatric disorder and one of the most disabling medical disorders [1]. As a mental illness OCD is associated with high rate of functional impairment, disability, caregiver burden and adverse psychosocial functioning [2-6]. People's perception of mental illness depends on the cultural background, level of education, beliefs in health, attitudes and trust in health care systems [7].

Studies suggest that despite having symptoms, many OCD patients do not seek treatment and continue to suffer [8]. This could be due to ignorance, stigma or different perceptions linked to socio-cultural traditions and religious beliefs. In Indian setting, OCD symptoms are prominently observed in some Hindu Bengali widows (characterized by repetitive washing and cleaning and purity rituals) which is shaped by religious traditions [9]. In a recent study conducted in India, majority of OCD patients attributed their illness and related suffering to fate or Karma theory which states that sufferings are brought due to nature of deeds in this or previous life [10] and is not "will of God" [11,12]. Thus, religious and tradition belief system plays a role in shaping attitude of people and patients towards OCD and health-care seeking behavior. Though studies

\footnotetext{
${ }^{1}$ Research Scholar, Department of Psychology, University of Calcutta, 92, A. P. C. ROAD, Kolkata, India

${ }^{2}$ Associate Professor, Department Of Psychology, University Of Calcutta, 92, A. P. C. Road, Kolkata, India *Responding Author

(C) 2016, R Das, S Raychaudhuri; licensee IJIP. This is an Open Access Research distributed under the terms of the Creative Commons Attribution License (http://creativecommons.org/licenses/by/2.0), which permits unrestricted use, distribution, and reproduction in any Medium, provided the original work is properly cited.
} 


\section{Disease Severity and Social Adjustment of Patients with Obsessive Compulsive Disorder from West Bengal}

on various psychiatric illnesses exist in Indian context, but detailed studies on OCD in Indian scenario are sparse.

In the present study we investigated severity of OCD with adjustment skills in patients diagnosed with OCD from the state of West Bengal covering rural, semirural and urban population.

\section{METHODS}

Referred OCD patients were considered across population groups: Urban, Semi-rural and rural (based on the residential address of patient). From the outpatient services (OPD) of government hospitals and private clinics by consultant psychiatrist patients were chosen after they satisfied the ICD 10 criteria (WHO, 1992) for diagnosis of OCD. YBOCS scale was used then for the clinical interview and selection of the patients.

Inclusion criteria involved: i) subjects diagnosed as suffering from OCD by a consultant psychiatrist, visiting the Psychiatric OPD of local medical colleges and hospitals or referred from clinics; ii) age range between 16-45 years for both genders; iii) naïve subjects i.e ones who are not acquainted with is kind of testing were chosen; iv) those willing to participate after being briefed about the purpose of the research.

The exclusion criteria involved: i) those who have history of any kind of psychiatric illness prior to onset of OCD; ii) those with psychiatric co-morbidity except depression; iii) those having any kind of physical illness iv) people with physical and mental disability; v) people with history of epilepsy; vi) those with history of head injury and vii) individuals with history of substance dependence or abuse and viii) individuals with history of developmental disorders.

\section{Data collection:}

The following forms were used for collecting data- 1) information schedule (patient information including case history), 2) Yale Brown Obsessive Compulsive Scale (YBOCS) 3) Rosenzweig Picture Frustration Test 4) and General Health Questionnaire GHQ-28.

Information schedule: This is the form we used to collect the information or case history of individual patients. It includes the basic information - name, age, gender, address, area of residence, religion/caste, mother tongue, educational level, occupation, marital status, family type, number of family members and patient's income. Each patient's presenting complaints as narrated by him/her and presented by informant were noted. Patients' pre-morbid personality along with mental status was also recorded. Information included religiosity (belief in religious values \& rituals) stated by patient or informant. 


\section{Disease Severity and Social Adjustment of Patients with Obsessive Compulsive Disorder from West Bengal}

Yale Brown Obsessive Compulsive Scale (YBOCS): We used the Y-BOCS scale to assess obsessions and compulsions. The range of severity for patients was categorized as: subclinical (0-7), Mild (8-15), Moderate (16-23), Severe (24-31) and Extreme (32-40). The Y-BOCS scale is independent of the specific nature of symptoms like counting or checking or washing, but is based on aspects of the symptoms as revealed by the patient during interview such as duration or interference, degree of resistance, etc. The scale is divided into two subscales: Obsession and Compulsion. For each subscale, five aspects of pathology are rated using scales ranging from 04: i) time spent, ii) interference, iii) distress from obsessions and iv) resistance to obsessions and v) control over obsessions. Y-BOCS total score is obtained as a sum of scores from both the subscales [13].

Rosenzweig Picture Frustration test: Rosenzweig PF test (Adult form) was administered to each patient with consent. The picture frustration study was developed as a result of experiments with repression and frustration carried out by Rosenzweig [14]. Based on the theory of frustration developed by himself, Rosenzweig compiled the "Picture Association study for accessing reactions to frustration", which later came to be known as Picture Frustration study (PF study). We have used the Indian adaptation in the present study [15].

General Health Questionnaire GHQ-28: The GHQ is a self administered screening test, which is sensitive to the presence of psychiatric disorders in individuals presenting in primary care settings and non- psychiatric clinical settings [16]. The GHQ is not designed to detect symptoms that occur with specific psychiatric diagnoses such as psychotic disorders, rather, provide a measure of overall psychological health or wellness. In order to assess this, the GHQ focuses on two major classes of phenomena: i) inability to continue to carry out normal "healthy" functions

and ii) symptoms of a distressing nature. There are several versions of the GHQ. GHQ-28 is the scale which is administered over here. Translated Bengali version of the scale is used here.GHQ-28 provides four specific subscales: somatic symptoms, anxiety and insomnia, social dysfunction and severe depression. It is important to note that these subscales do not necessarily correspond to psychiatric diagnosis nor are the subscales independent of each other.

\section{Statistical analyses}

We analyzed data using Epi Info software [17] which is widely used for statistical analyses of medical and epidemiological data.

\section{RESULTS}

Socio-demographic data:

A total of 201 OCD patients were considered in the study. The patients were divided into three populations based on the area of residence: Urban, Semirural and Rural. In the urban population $58.7 \%$ were males and $41.3 \%$ females. The semirural population group consisted of $48 \%$ males 


\section{Disease Severity and Social Adjustment of Patients with Obsessive Compulsive Disorder from West Bengal}

and 52\% females and the rural population group consisted of 54.2\% males and $45.8 \%$ females. Except one all other patients were Hindu by religion. Details are summarized in Table 1.

\section{Severity of OCD based on Y-BOCS:}

In the present study YBOCS scale is used for the assessment of OCD severity. Comparison of OCD severity between the different population was carried out and statistics based on YBOCS score is summarized in Table 2.

Comparing Rural, Semirural and Urban populations, by Bartlett's test for inequality of population variances (Chi-squared $=19.34$, degrees of freedom $=2$ and $\mathrm{P}$ value $=0.001$ ) revealed that the variances were not equal. Hence, Kruskal-Wallis test (equivalent of ANOVA) for comparison of three populations was conducted (Kruskal-Wallis $\mathrm{H}=2.33$, degrees of freedom $=$ 2 and P-value $=0.312$ ). Since P-value is greater than $5 \%$, it is inferred that the mean YBOCS scores are not significantly different in all three populations.

Comparison between rural and semirural populations based on YBOCS scores (Table 2) does not reveal any significant difference (P-value $=0.576$, $\mathrm{t}$-value $=-0.5609$, T-Test). No significant difference in the YBOCS scores between Urban and Rural population was observed ( $\mathrm{T}$-value= 0.502 , P-value $=0.616$, T-test). Similarly, no significant difference in the YBOCS scores between Semi-rural and Urban population was found $(\mathrm{T}$-value $=-0.531$, P-value $=0.592$, T-test $)$.

Majority of the rural population, 40 out of 59 were categorized as 'severe', considerable proportion as 'moderate' 15 and very few 3 in 'extreme' and only 1 in 'mild' category. Of 92 patients residing in urban areas 17 were 'extreme', 41 had 'severe', 30 had 'moderate' and only 4 had 'mild' OCD. Of the 50 patients of the 'semirural' population, 5 were categorized as 'extreme', 33 'severe', 9 'moderate' and only 1 'mild'. There were 2 patients having YBOCS score 7 and were considered as borderline.

\section{Distribution of OCD disease severity and GHQ-28 score in Urban, Semirural and rural populations}

The GHQ-28 questionnaire (Translated in Bengali) was provided to each patient, since all declared Bengali as the mother tongue.

Since the range of GHQ score is 0 - 28, we divided the patients into two groups , one with GHQ score $<14$ and the other with GHQ score $\geq 14$, where 14 is the halfway mark. The distribution of disease severity and GHQ-28 score in Urban, Semirural and rural populations is summarized in Table 3. 


\section{Disease Severity and Social Adjustment of Patients with Obsessive Compulsive Disorder from West Bengal}

It is observed that for each population group, members of each YBOCS category are almost equally distributed between the two classes of GHQ i.e, 'GHQ score $<14$ ' and 'GHQ score $\geq$ 14 '. Hence, no correlation could be ascertained between high GHQ score $(\geq 14)$ and severity of OCD.

\section{Analysis of Rosenzweig PF test}

Group Conformity Rating (GCR) obtained in the Rosenzweig PF test is "one measure of the individual's adjustment to a normal group $[15,18]$, which reflects adjustment of individual to society. High GCR score correspond to low frustration and adequate adjustment to normal group. Low GCR score below 40 usually confers to high level of frustration and poor adjustment to surroundings and normal group / society.

[URL: shodhganga.inflibnet.ac.in/bitstream/10603/33293/10/10_chapter\%205.pdf].

In the urban population $44.5 \%$ of patients were inadequately adjusted to peer group / society and $30.4 \%$ were poorly adjusted, whereas $15.2 \%$ and $9.7 \%$ were moderately and adequately adjusted respectively. In the semirural population $48 \%$ were inadequately adjusted, $42 \%$ of patients were poorly adjusted to society, whereas $4 \%$ and $6 \%$ were moderately and adequately adjusted respectively. In the rural population $37.2 \%$ were inadequately adjusted, $23.7 \%$ of patients were poorly adjusted to peer group / society, whereas $13.5 \%$ and $25.4 \%$ were moderately and adequately adjusted. Thus, in the rural population a quarter of patients is well adjusted to peer group in spite of disease which is in contrast to the scenario in Urban and semirural populations.

\section{Religiosity and severity of OCD:}

Religiosity of the individual patient was revealed in the personal interview and recorded in the information schedule. In some cases patient himself / herself revealed or declared religiosity (belief in religious values \& rituals) and in others accompanying persons (informants) described existence of religiosity in patients.

In the rural population 31 patients out of 43 (belonging to 'extreme' and 'severe' YBOCS category) had evidence of religiosity and were practicing rituals. Out of 15 patients suffering from 'moderate' and 'mild' OCD only 4 had evidence of religiosity.

In the semirural population, 25 patients out of 38 (with 'extreme' and 'severe' OCD) had evidence of religiosity. Out of 9 patients suffering from 'moderate' and 'mild' OCD only 3 reported religiosity.

Overall, highest number of patients (extreme + severe OCD) with religiosity occurs in rural (59\%) followed by semi-rural (56\%) and urban (37\%) populations. 


\section{Disease Severity and Social Adjustment of Patients with Obsessive Compulsive Disorder from West Bengal}

\section{DISCUSSIONS}

In the present study we assessed 201 OCD patients from West Bengal covering rural, semirural and urban areas in the age range of $16-45$ yrs. The urban and rural populations consisted of more males than females, which is consistent with trend that males had higher rate than women as reported in epidemiological studies [19, 20]. However, the semirural population consisted of more females. This may be due to the fact that more females came for treatment and by chance. The study is not a survey but relies on data collected from patients reporting to hospitals and clinics.

Evaluation of severity of OCD was carried out on the basis of Y-BOCS scores. Comparative analyses of score from populations rural, semirural and urban did not reveal statistically significant differences. Similarly comparison of rural vs. semirural, rural vs. urban and semirural vs urban did not reveal any statistically significant differences. This signifies that the distribution of OCD severity is similar for all populations.

Rural and semirural populations had the largest percentage of severe cases (68\% and $66 \%$ respectively). About 32\% of the urban patients had moderate OCD compared to semirural $(\sim 18 \%)$ and rural ( $25 \%)$. Urban population had the largest share of extreme cases. That urban population has more OCD severity is similar to a recent finding reported from Warangal region of Andhra Pradesh state in Southern India [21].

For each patient the general health was evaluated using the GHQ-28 protocol. No association was found between high GHQ score (impaired health and functioning) and severity of OCD for all populations.

On the basis of the findings of Rosenzweig analyses we found that in urban and semirural populations majority of patients ( $75 \%$ and $\sim 90 \%$ respectively) had inadequate or poor adjustment to society and surroundings. In contrast, the rural population had the largest proportion of patients (25.4\%) who are highly adjusted.

It should also be mentioned that majority of patients (> 56\%) with severity of OCD ('extreme' + 'severe' cases) from rural and semi-rural areas had religiosity (strong belief in religious traditions with performance of rites and rituals). In the urban patients, only a third of the 'extreme' + 'severe' cases reported religiosity. That OCD is linked with religiosity is known in different societies across the world [22-24]. In our study we are finding similar trends.

\section{CONCLUSION}

The present study was limited to a few hospitals and clinics in West Bengal and involved patients referred thereof. The samples do not represent a survey. Hence, in reality this data does 


\section{Disease Severity and Social Adjustment of Patients with Obsessive Compulsive Disorder from West Bengal}

not account for true community prevalence. The true rate of OCD and manifestations of features in community may be much higher than estimated in the study.

Overall, prevalence of OCD was found more in the age group 25 - 40 yrs with the mean age being 35 yrs across populations from rural, semirural and urban areas. More males were affected than females. Majority of OCD patients belonged to urban areas. Not all patients with severe OCD had religiosity, though majority had beliefs and practiced rites and rituals. The study being hospital based does reflect prevalence but does not represent the true prevalence rates in the population of West Bengal. Hence, detailed survey based studies may be required in future.

\section{Acknowledgments}

The author appreciates all those who participated in the study and helped to facilitate the research process.

\section{Conflict of Interests}

The author declared no conflict of interests.

\section{REFERENCE}

1. Weissman, MM., Bland, RC., Canino, GJ., et al. (1994) The cross national epidemiology of obsessive compulsive disorder. J Clin Psychiatry, 55: ( suppl.) : 5-10.

2. Rasmussen, SA., Eisen, JL. (1992) The epidemiology and clinical features of obsessive compulsive disorder. Psychiatric Clinics North America, 15: 743-758.

3. Vikas, A., Avasthi, A., Sharan, P. (2011) Psychosocial impact of obsessive- compulsive disorder on patients and their caregivers: a comparative study with depressive disorder. International J Social Psychiatry, 57: 45-56.

4. Grover, S., Dutt, A. (2011) Perceived burden and quality of life of caregivers in obsessive- compulsive disorder. Psychiatry Clinical Neurosciences, 65: 416- 22.

5. Chakraborti, S., Kulhara, P., Verma SK. (1993) The pattern of burden in families of neurotic patients. Social Psychiatry Psychiatric Epidemiology, 28: 172-177.

6. IDEAS (Indian Disability Evaluation and Assessment Scale)- (2002) A scale for measuring and quantifying disability in mental disorders, Indian Psychiatric Society.

7. Ekanayake, S., Ahmed, F., Mc Kenzie, K. (2012) Qualitative cross-sectional study of the perceived causes of depression in South Asian origin women in Toronto. BMJ Open, 2:e000641.

8. Fireman, B., Koran, LM., Leventhal, JL., et al. (2001) The prevalence of clinically recognized obsessive-compulsive disorder in a large health maintenance organization. American J Psychiatry, 158: 1904-10.

9. Chakraborty, A., Banerji, G. (1975) Ritual, a culture Specific Neurosis and Obsessional States in Bengali Culture. Ind J Psychiatry, 17: 273-280. 


\section{Disease Severity and Social Adjustment of Patients with Obsessive Compulsive Disorder from West Bengal}

10. Patra, BN., Grover, S., Aggarwal, M., Avasthi, A., Chakrabarti, S., Malhotra, S. (2013) Explanatory models in patients with obsessive compulsive disorder: an exploratory study. Ind J Social Psychiatry, 29(1-2): 38 -45.

11. Varma, VK. (2009) Ancient Indian concepts of reality, causality and cosmology. In : Varma, VK., Kala, AK., Gupta, N., (Eds.). “Culture, personality and mental illness”. Jaypee Brothers, New Delhi, Page: 5-22.

12. Varma, VK. (2009) Culture and Indian personality. In : Varma, VK., Kala, AK., Gupta, N., (Eds.). “Culture, personality and mental illness”. Jaypee Brothers, New Delhi, Page: 177-187.

13. Goodman, WK., Price, LH., Rasmussen, SA., et al. (1989) The Yale-Brown ObsessiveCompulsive Scale. I. Development, Use, and Reliability. Arch. Gen. Psychiatry, 46: 1006-1011.

14. Rosenzweig, S. (1943) An Experimental study of "repression” with special reference to need persistive and ego defensive reactions to frustration. J Expo. Psychol., 32: 64-74.

15. Rosenzweig PF Study Adult Form. Indian Adaptation. Edition 2010. U Pareek, Devi RS et al. Prasad Psycho Corporation.

16. Goldberg, DP., Hillier, VF. (1979) A scaled version of the General Health Questionnaire. Psychol Med., 9(1): 139-45.

17. Epi Info. Division of Health Informatics \& Surveillance (DHIS), Center for Surveillance, Epidemiology \& Laboratory Services (CSELS), CDC Atlanta, US.

18. Keertish, N., Sharma, I. (2015) Study of Frustration in Adolescents with Conversion Disorder. J. Indian Assoc. Child Adolesc. Ment. Health, 11(1): 7-31.

19. Fatemeh-Assarin, MD., HosseinBiqam, MA., AsqarAsqarmejad. (2006) An epidemiological study of obsessive compulsive disorder among high school students and its relationship with religious attitudes. Arch Iranian Med., 9: 104-107.

20. Heyman, I., Fombonne, E., Simmons, H., et al. (2001) Prevalance of obsessive compulsive disorder in the Britiish Nationwide survey of child mental health. $\mathrm{Br} \mathrm{J}$ Psychiatry, 179: 324-329.

21. Revoori, SR., Peddi, SK., Alladi, M. (2016) An epidemiological study and severity assessment of obsessive compulsive disorder in Warangal region, India. Eur J Pharm Med Res., 3(4): 205-210.

22. Abramowitz, JS., Deacon, BJ., Woods, CM., Tolin, DF. (2004) Association between Protestant religiosity and obsessive- compulsive symptoms and cognitions. Depress Anxiety, 20(2): 70-76.

23. Cenk, T., Ulug, B. (2001) Religiosity and religious obsessions in obsessive compulsive disorder. Psychiatry Res., 104(2): 99-108.

24. Yorulmaz, O., Gencoz, T., Woody, S. (2009) OCD cognitions and symptoms in different religious contexts. J Anxiety Disorders, 23(3): 401-406. 
Disease Severity and Social Adjustment of Patients with Obsessive Compulsive Disorder from West Bengal

Table 1: Details of socio-demographic information

\begin{tabular}{|c|c|c|c|c|}
\hline & & $\begin{array}{l}\text { Rural } \\
(n=59)\end{array}$ & $\begin{array}{l}\text { Semirural } \\
(n=50)\end{array}$ & $\begin{array}{l}\text { Urban } \\
(\mathrm{n}=92)\end{array}$ \\
\hline \multicolumn{2}{|l|}{ Male (\%) } & $32(54.2 \%)$ & 24 (48\%) & 54 (58.7\%) \\
\hline \multicolumn{2}{|l|}{ Female (\%) } & 27 (45.8\%) & 26 (52\%) & 38 (41.3\%) \\
\hline \multicolumn{2}{|l|}{ Mean Age (S.D) } & $35.3(7.3)$ & 34.7 (8.6) & $34.2(8.7)$ \\
\hline \multirow[t]{2}{*}{ Religion } & Hindu & 59 & 50 & 91 \\
\hline & Other & & & 1 \\
\hline
\end{tabular}

Table 2: Statistics on YBOCS score - Comparison of Rural, Semi-rural and Urban population

\begin{tabular}{|l|l|l|l|l|}
\hline Population & $\begin{array}{l}\text { Number of } \\
\text { observations }\end{array}$ & Mean & SD & Median \\
\hline Rural & 59 & 25.6 & 3.6 & 26.0 \\
\hline Semirural & 50 & 26.2 & 6.4 & 27.0 \\
\hline Urban & 92 & 26.0 & 5.9 & 26.0 \\
\hline
\end{tabular}

Table 3: Distribution of Y-BOCS category in different population groups

\begin{tabular}{|l|l|l|l|}
\hline Y-BOCS Category & $\begin{array}{l}\text { Rural } \\
(\mathbf{n = 5 9 )}\end{array}$ & $\begin{array}{l}\text { Semirural } \\
(\mathbf{n = 5 0 )}\end{array}$ & $\begin{array}{l}\text { Urban } \\
(\mathbf{n = 9 2})\end{array}$ \\
\hline Extreme & $3(5 \%)$ & $5(10 \%)$ & $17(18.5 \%)$ \\
\hline Severe & $40(67.7 \%)$ & $33(66 \%)$ & $41(44.5 \%)$ \\
\hline Moderate & $15(25.4 \%)$ & $9(18 \%)$ & $30(32.6 \%)$ \\
\hline Mild & $1(1.6 \%)$ & $1(2 \%)$ & $4(4.3 \%)$ \\
\hline Borderline/Subclinical & 0 & $2(4 \%)$ & 0 \\
\hline
\end{tabular}

Table 4: Distribution of OCD disease severity and GHQ-28 score in Urban, Semirural and rural populations.

\begin{tabular}{|l|c|c|c|}
\hline & Y-BOCS & GHQ score $<\mathbf{1 4}$ & GHQ score $\geq \mathbf{1 4}$ \\
\hline Urban (n=92) & \multicolumn{3}{|c|}{} \\
\hline & Extreme & 6 & 11 \\
\hline & Severe & 22 & 19 \\
\hline & Moderate & 16 & 14 \\
\hline Semi-rural (n=50) & Mild & 2 & 2 \\
\hline & Extreme & 0 & 16 \\
\hline & Severe & 17 & 5 \\
\hline & Moderate & 4 & 0 \\
\hline & Mild & 1 & - \\
\hline & Borderline & 2 & \\
\hline
\end{tabular}

(C) The International Journal of Indian Psychology, ISSN 2348-5396 (e)| ISSN: 2349-3429 (p) | 89 
Disease Severity and Social Adjustment of Patients with Obsessive Compulsive Disorder from West Bengal

\begin{tabular}{|l|c|c|c|}
\hline & Y-BOCS & GHQ score $<\mathbf{1 4}$ & GHQ score $\geq \mathbf{1 4}$ \\
\hline Rural $(\mathrm{n}=59)$ & & 2 & 1 \\
\hline & Extreme & 27 & 13 \\
\hline & Severe & 8 & 7 \\
\hline & Moderate & 1 & 0 \\
\hline
\end{tabular}

Table 5: Analysis on Rosenzweig PF test.

\begin{tabular}{|c|c|c|c|c|}
\hline & & $\begin{array}{l}\text { Urban } \\
(n=92)\end{array}$ & $\begin{array}{l}\text { Semi-Rural } \\
\quad(n=50)\end{array}$ & $\begin{array}{l}\text { Rural } \\
(n=59)\end{array}$ \\
\hline Male (\%) & & $54(58.7 \%)$ & $24(48 \%)$ & $32(54.2 \%)$ \\
\hline Female (\%) & & 38 (41.3\%) & $26(52 \%)$ & $27(45.8 \%)$ \\
\hline Mean Age (S.D) & & $34.2(8.7)$ & 34.7 (8.6) & $35.3(7.3)$ \\
\hline Mean Rosenzweig GC & score (S.D.) & $53.9(12.24)$ & $49.6(12.7)$ & $59.2(15.7)$ \\
\hline Rosenzweig & Poor (\%) & $28(30.4 \%)$ & $21(42 \%)$ & $14(23.7 \%)$ \\
\hline Category* & Inadequate (\%) & $41(44.5 \%)$ & $24(48 \%)$ & $22(37.2 \%)$ \\
\hline (Adjustment with peer & Moderate (\%) & $14(15.2 \%)$ & $2(4 \%)$ & $8(13.5 \%)$ \\
\hline group) & Adequate (\%) & $9(9.7 \%)$ & $3(6 \%)$ & $15(25.4 \%)$ \\
\hline Religion & Hindu & 91 & 50 & 59 \\
\hline & Other & 1 & 0 & 0 \\
\hline
\end{tabular}

$*$ N.B. Rosenzweig GCR score as measure of social adjustment : 0 - $40 \rightarrow$ Poor; 41 - $60 \rightarrow$ Inadequate; 61 - $70 \rightarrow$ Moderate; 71 onwards: High (Adequate).

How to cite this article: R Das, S Raychaudhuri (2016), Disease Severity and Social Adjustment of Patients with Obsessive Compulsive Disorder from West Bengal, International Journal of Indian Psychology, Volume 3, Issue 4, No. 63, ISSN 2348-5396 (e), ISSN: 2349-3429 (p), DIP: 18.01.102/20160304, ISBN: 978-1-365-32518-2 